\title{
Strates
}

STRATES Matériaux pour la recherche en sciences sociales

$10 \mid 2001$

Villageois et citadins de Grèce

\section{Le village et la ville : la Grèce imaginaire dans les manuels scolaires du $\mathrm{XX}^{\mathrm{e}}$ siècle}

\section{Christina Koulouri}

\section{(2) OpenEdition \\ 1 Journals}

\section{Édition électronique}

URL : http://journals.openedition.org/strates/48

DOI : $10.4000 /$ strates.48

ISSN : $1777-5442$

\section{Éditeur}

Laboratoire Ladyss

\section{Édition imprimée}

Date de publication : 1 mai 2001

ISSN : 0768-8067

\section{Référence électronique}

Christina Koulouri, « Le village et la ville : la Grèce imaginaire dans les manuels scolaires du XXe siècle », Strates [En ligne], 10 | 2001, mis en ligne le 11 janvier 2005, consulté le 08 septembre 2020. URL : http://journals.openedition.org/strates/48; DOI : https://doi.org/10.4000/strates.48

Ce document a été généré automatiquement le 8 septembre 2020

Tous droits réservés 


\title{
Le village et la ville : la Grèce imaginaire dans les manuels scolaires du $\mathrm{XX}^{\mathrm{e}}$ siècle
}

\author{
Christina Koulouri
}

Les contradictions des manuels scolaires contemporains

Chaque village a son histoire. Pierre a appris l'histoire de son village et il la raconte : dans les temps anciens, ce lieu n'était pas habité. Il était couvert de sapins et les eaux y étaient abondantes. Il y avait un seul pommier. Un jour, des gens sont arrivés et ils ont dit: "Comme c'est beau! Construisons ici nos maisons. " Ainsi furent bâties les premières maisons. Puis d'autres ont été construites et elles ont formé un village. On l'a appelé Milia (pommier). La vie des habitants était difficile. La rivière débordait et provoquait des catastrophes. En hiver la neige les coupait du monde. Les animaux sauvages dévoraient leurs troupeaux. Des brigands ravissaient leurs biens. Ils travaillaient dur pour gagner leur pain : certains étaient bergers, d'autres agriculteurs ou bûcherons... D'autres devaient émigrer. [...] Peu à peu, beaucoup de choses ont changé dans le village et la vie s'est améliorée. Aujourd'hui, nous avons des rues, une école, l'électricité, une clinique... Nous avons aussi fondé une coopérative pour protéger la forêt et gagner plus d'argent ${ }^{1}$.

Ce passage d'un manuel contemporain "d'étude de l'environnement» destiné à la deuxième année de l'école primaire ${ }^{2}$ esquisse l'image positive de la Grèce rurale contemporaine véhiculée par un certain nombre de manuels scolaires. Dans cette représentation, la campagne grecque a bénéficié des progrès techniques et scientifiques, elle s'est modernisée, la vie de ses habitants est devenue plus aisée. La description rétrospective des changements dans le village et en Grèce rurale inspire optimisme et confiance dans le progrès.

2 Pourtant, dans la même classe de primaire, le livre de lecture donne une image diamétralement opposée :

Après le tournant, le village est apparu. Ses maisons semblaient collées les unes aux autres, l'église était au centre, la petite école à la périphérie... Dans la rue, personne. La solitude régnait. Pas un animal, pas une voix, pas la moindre fumée sortant d'une cheminée. Un chien marchait, seul, la tête baissée, dans un chemin étroit et sombre... Catherine regardait les maisons. Les fenêtres étaient fermées, 
certaines avaient des volets cassés. Les murs n'avaient pas été crépis depuis de longues années, les toits tombaient en ruine. La jeune fille sentit son cœur se serrer. Soudain, dans une rue vide, elle crut voir quelque chose bouger, une ombre. Une vieille dame en noir apparut. Elle était bossue, s'appuyait sur un bâton noueux, son visage était ridé. "Tout le monde est parti ", dit-elle. "Tout le monde. Personne n'est resté. Ils ont tout abandonné et ils sont partis... » Et elle s'en alla lentement, comme elle était venue, appuyée sur son bâton noueux ${ }^{3}$.

3 L'exode rural, l'abandon de la campagne grecque et le vieillissement de sa population, telle est la réalité décrite par ce texte. Le récit est empreint de mélancolie et de pessimisme, la campagne est désertée, privée de vie et d'espoir.

Une troisième catégorie de textes rencontrés dans des manuels scolaires contemporains évoque le paradis perdu, une vie simple près de la nature, riche de joies quotidiennes et d'affection :

Je suis né et j'ai passé mon enfance dans un petit village montagnard. Mon village était pauvre et nous étions pauvres, mais personne n'y prêtait attention. On jouait du matin au soir dans la campagne, on se promenait en forêt, on grimpait aux arbres, c'était tout ${ }^{4}$.

5 Le souvenir nostalgique du village s'oppose en général à l'expérience de la vie en ville (en particulier à Athènes), marquée par le stress quotidien et le manque de communication entre les hommes. Cette représentation est complétée par celle du village comme lieu de villégiature. Dans les livres de lecture, on rencontre fréquemment des enfants qui racontent leur séjour dans un village, à la montagne ou dans une île, qui est souvent le village natal de leurs parents.

Théodore a passé l'été dans le village de son père. Il avait emporté un petit magnétophone [...] qui a passionné les enfants. Ils en ont fait un jeu, en enregistrant et en écoutant tout ce qu'on peut imaginer. - Allons dans la basse-cour, disait l'un. Allons voir les petits cochons de la mère Stamatina, disait l'autre.[...] À la fin des vacances, Théodore a emporté la cassette. Quand il se lève le matin, il l'insère souvent dans le magnétophone et la maison résonne des voix de la campagne. Ses parents le grondent : - Ne mets pas toujours la même chose, Théodore ! Moins fort ! On va nous chasser de l'immeuble. - Tant mieux ! dit Théodore en riant. Qu'on nous chasse, nous irons au village ${ }^{5}$ !...

6 La lecture des textes cités, qui illustrent des aspects différents de la campagne grecque, suscite plusieurs questions. On pourrait d'abord supposer que l'image de la Grèce rurale véhiculée par les manuels scolaires est un reflet fidèle de la réalité et que ces manuels peuvent donc servir de source complémentaire pour étudier le monde rural grec. Il est vrai que ces manuels sont, dans une certaine mesure, le reflet de la société qui les produit - ou du moins de certaines de ses composantes, en position dominante. Ils ne peuvent pourtant pas être considérés comme un reflet fidèle : du fait des inerties de l'institution scolaire, l'introduction de nouveaux éléments dans le discours scolaire s'opère avec un relatif retard et les manuels n'intègrent que rarement l'innovation et le changement ; par ailleurs, le rôle édifiant de ces manuels est particulièrement marqué en Grèce, où l'État a le monopole de leur production et de leur diffusion gratuite dans les écoles, et où règne le système du manuel unique par classe et par matière ${ }^{6}$.

On peut donc se demander dans quelle mesure l'image proposée par ces manuels est fictive ou conforme à la réalité, et analyser, en particulier, le rapport entre les transformations de l'économie et de la société grecque tout au long du $\mathrm{XX}^{\mathrm{e}}$ siècle, et les représentations de ces transformations dans le discours scolaire. Dans le cas précis de la Grèce rurale, on peut étudier comment elle est décrite et évaluée, et quels sont ses 
rapports avec la Grèce urbaine: par exemple, noter si le récit se déroule dans un environnement urbain ou rural, si le village est défini ou apprécié en opposition à la ville, etc.

8 Pour interpréter le discours scolaire de façon cohérente, la connaissance de la réalité sociale est évidemment indispensable. Selon les données statistiques, la population rurale a diminué de façon considérable au cours du vingtième siècle, passant de $61,9 \%$ de la population totale en 1920 à $30,3 \%$ en $1981^{7}$. Ce processus s'est accéléré dans les années 1961-19718, quand l'exode rural a nourri l'urbanisation et le développement disproportionné de la capitale grecque. La chute de la population rurale et le passage progressif à la société industrielle ont entraîné inévitablement la disparition du mode de vie traditionnel et l'émergence d'une culture urbaine qui s'est imposée comme culture nationale. Les élèves résidant désormais plutôt dans des centres urbains que dans de petits villages, les récits des manuels scolaires auraient dû se situer aussi dans l'espace urbain pour que les lecteurs puissent s'y reconnaître.

9 En fait, les manuels n'ont pas toujours tenu compte des transformations démographiques, économiques et sociales. Des descriptions idéalisantes et réalistes alternent ou parfois coexistent. Ces fluctuations du discours scolaire dépendent largement, on le verra, du contexte historique et plus particulièrement du régime politique.

Ce travail s'appuie sur deux types de manuels scolaires: les livres de lecture et les manuels scolaires de géographie ${ }^{9}$. Les raisons de ce choix sont simples. La discipline qui enseigne par excellence les paramètres de l'évolution du monde rural et urbain est la géographie. Mais le livre de lecture, qui résume les connaissances et les valeurs qu'on se propose de transmettre aux jeunes générations, s'avère un complément indispensable pour une telle analyse. L'importance du livre de lecture est d'autant plus grande que les manuels de géographie ne sont souvent que de sèches énumérations de données statistiques, qui ne sont ni interprétées ni commentées.

11 D'une façon générale, les livres de lecture sont conçus selon deux modèles : soit ils se fondent sur un récit continu comportant un ou plusieurs personnages principaux, soit ils comprennent des histoires indépendantes les unes des autres. Dans le premier cas, on peut noter si l'histoire se déroule à la campagne ou en ville, et quelle est l'image produite par le texte ou par l'illustration. En revanche, dans le second cas, la majorité des textes ne sont pas clairement situés dans le temps et dans l'espace - qu'il s'agisse d'histoires d'animaux, d'adultes ou d'enfants. Ce type de récit permet de formuler des règles générales de conduite et d'offrir aux enfants des modèles édifiants atemporels.

Le réalisme optimiste de l'entre-deux-guerres

12 Le livre de lecture de Galateia Kazantzaki Le jeune agriculteur (1921) relève du premier modèle et, comme le révèle son titre, il se déroule entièrement dans la Grèce rurale. L'histoire, située dans un "village béni ", apporte des connaissances en histoire et en histoire naturelle, des recommandations relatives à l'hygiène et des leçons édifiantes. L'image de la vie au village est manifestement idéalisée, ce qui est le cas pour la plupart des manuels scolaires parus dans les années vingt.

Ce lieu est béni! Les hommes y travaillent la terre avec patience, et la terre les récompense avec prodigalité. - Comme la terre est généreuse! disait un vieux paysan émerveillé par l'abondance des fruits. Elle rend tout au décuple. La sueur humaine est de l'eau bénite là où elle tombe.[...] Dans ce village, les gens étaient gais et sains ${ }^{10} \ldots$ 

monde $^{14}$ ».

Les manuels scolaires de l'entre-deux-guerres sont généralement optimistes. Alors qu'une crise affecte presque tous les aspects de la vie publique, ils s'efforcent d'offrir une représentation positive de l'avenir collectif. Tout en véhiculant des images idylliques du village grec habité par des paysans laborieux et bienveillants, ils plaident en faveur de la modernisation de l'économie rurale par le bais du progrès technique. On y trouve d'ailleurs souvent des conseils pour améliorer les méthodes de l'agriculture et de l'élevage, qui incitent les élèves à croire au progrès technique et à adopter l'innovation, dans l'intérêt national.

Ces manuels scolaires de l'entre-deux-guerres sont les héritiers du courant littéraire de l'éthographie qui s'est épanoui à partir de la fin du XIXe siècle, caractérisé par une idylle rurale, des scènes de la vie paysanne, un récit réaliste et une intrigue simple ${ }^{11}$. L'éthographie grecque, marquée par un "réalisme rêveur ou bâillonné12 ", allait de pair avec le courant progressiste de "démoticisme » (démotiki = langue populaire, parlée) qui revendiquait l'introduction de la langue parlée dans l'enseignement et valorisait la culture néo-hellénique au détriment de l'admiration pour la Grèce ancienne. Le paysan simple et pur de la campagne grecque devint le héros de ce courant, ses mœurs et sa vie furent exaltées et décrites minutieusement. Aux yeux des démoticistes, c'était lui, et non Périclès, qui symbolisait le caractère national. Il faut souligner l'importance de ce tournant idéologique après un siècle d'admiration exclusive pour la gloire de la Grèce ancienne qui impliquait le mépris pour le présent et la Grèce moderne.

Simultanément, les manuels scolaires des années vingt délivraient, on l'a dit, un message moderniste. Les intellectuels libéraux, dont certains étaient aussi des auteurs de manuels scolaires, aspiraient à une réforme profonde de tous les aspects de la vie publique. Il n'est donc pas étonnant que dans les manuels de cette période abondent les recommandations et les attentes. Par exemple, on lit dans un manuel de géographie publié vers la fin des années vingt:

Quand les marais et les lacs de Macédoine orientale seront asséchés, nous obtiendrons des plaines vastes et fertiles qui produiront du blé en abondance, du riz et du coton permettant à la Grèce d'être autosuffisante. Elles produiront aussi des betteraves sucrières. Les principales composantes de notre alimentation et de notre habillement seront ainsi produites par des régions aujourd'hui improductives et mortifères [...]. Il est facile de comprendre pourquoi la Grèce est pauvre ${ }^{13}$.

Les origines de cette position doivent être recherchées dans le grand courant politique et idéologique connu comme vénizélisme ( $\mathrm{du}$ nom d'El. Vénizélos, homme politique éminent et Premier ministre de 1910 à 1920 et de 1928 à 1932) qui s'impose en Grèce de 1910 à 1932. Ce courant, qui regroupe des gens de toutes classes sociales (grande bourgeoisie d'affaires, petite bourgeoisie, paysans sans terres qui vont bénéficier de la réforme agraire de 1917, réfugiés d'Asie Mineure après 1922) et qui touche tous les aspects de la vie publique, est l'agent de la modernisation bourgeoise du pays, selon le modèle occidental du capitalisme et de la république bourgeoise libérale. Au niveau idéologique, il se caractérise par « la fermeté, l'optimisme, le réalisme, l'attachement au concret, la clarté et en général tout ce qui implique la volonté de réforme raisonnée du

Dans les livres de lecture des années trente, la ville apparaît pour la première fois comme scène où le récit est situé - à côté du village qui occupe toujours la plus grande place. La ville est encore anonyme : on ne sait pas si les petits héros vivent à Athènes ou dans un bourg. Le récit laisse pourtant entendre qu'il y a un rapport étroit entre la ville 
et la campagne. Les habitants du village - y compris les enfants - partent pour travailler à la ville comme employés de commerce ou comme domestiques ${ }^{15}$.

Les manuels scolaires de l'entre-deux-guerres reflètent dans une certaine mesure la réalité sociale et économique, ainsi que les mentalités dominantes. La Grèce était alors confrontée à un grave problème démographique et économique produit par l'arrivée d'un million et demi de réfugiés après la guerre en Asie Mineure en 1922. La presse quotidienne engageait à développer l'industrie pour créer de nouveaux emplois, et à moderniser l'agriculture pour réduire l'exode rural ${ }^{16}$. Dans un premier temps, la maind'œuvre urbaine et industrielle provenait de la campagne, mais de façon saisonnière et temporaire. La famille restait au village et les hommes, ainsi qu'un nombre important de jeunes garçons, émigraient en ville ${ }^{17}$.

De 1949 à 1974 : la fausse harmonie et le refuge de la campagne " pure »

Le réalisme de l'entre-deux-guerres recule après la fin de la guerre civile (1946-1949). Juste après la fin de la seconde guerre mondiale, on trouve encore des descriptions réalistes, qui sont empreintes d'un pessimisme manifeste. Par exemple, en 1947, on peut lire dans un manuel de géographie :

Malheureusement l'agriculture n'est pas suffisamment développée, et en ce qui concerne certains produits, ses méthodes sont archaïques. L'élevage et l'exploitation forestière sont tout autant négligés. Même la pêche accuse un retard $^{18}$.

Mais, dans les années cinquante, ce sont les silences et les descriptions idéalisantes qui l'emportent. Dans des manuels scolaires de géographie, les auteurs préfèrent le discours «neutre " des chiffres et de la sèche énumération (population, superficie, production, etc. de chaque région) ainsi que de vagues descriptions ornées d'élégants adjectifs. Par exemple, Athènes est décrite de la façon suivante, dans un manuel datant de 1952 :

C'est une vaste étendue de maisons, séparées par des rues, larges, étroites ou de taille moyenne, dont la plupart sont asphaltées et où la circulation des voitures et des piétons est intense ${ }^{19}$.

21 Quant aux livres de lecture de la même période, ils ignorent la ville et situent leur récit dans la Grèce rurale, de façon plus ou moins précise. Si la ville est décrite, c'est à travers le regard du paysan: hors de portée du héros, elle apparaît comme quelque chose de merveilleux et d'exotique. Un des textes d'un livre de lecture destiné à la troisième année de primaire porte le titre éloquent : «Que la ville était différente ${ }^{20}$ ! ».

Le corpus que nous avons étudié pour la période d'après-guerre montre un décalage important entre la réalité sociale et le contenu des manuels. L'urbanisation croissante et l'émigration massive ne sont pas reflétées dans les livres de lecture qui, contrairement à la période de l'entre-deux-guerres, passent sous silence les problèmes de l'économie et de la société grecques et en donnent une image idéalisée. Le village grec est un lieu idyllique où les gens vivent heureux. La description de la campagne et des villes évoque des rédactions d'enfants: la beauté des paysages, la douceur du climat, les villes florissantes sont les éléments principaux du récit. Cette image vague ou harmonieuse vise à cacher une vie politique troublée, marquée par une profonde dichotomie héritée de la guerre civile.

La «Nouvelle Discorde" séparait alors les Grecs "patriotes» - de droite, voire d'extrême-droite - et les Grecs «non patriotes » - de gauche. Le «terrorisme légalisé ${ }^{1}$ »du pouvoir central, le rôle politique de la Couronne et de l'armée, les interventions de 
la Grande-Bretagne puis des États-Unis altéraient le parlementarisme républicain du régime. La crise politique n'était pas sans rapport avec la crise sociale due aux fortes inégalités de revenus et à l'ampleur de la pauvreté, du chômage et de l'émigration. Le pessimisme néo-réaliste du cinéma grec d'après-guerre fournit d'ailleurs des images révélatrices de cette crise multiforme. Au niveau idéologique, le pouvoir est alors confronté à une crise de légitimation qui traduit un sentiment généralisé d'insécurité. C'est à la lumière de cette réalité politique et sociale que nous devons considérer le contenu des manuels de cette période. Selon nous, la fuite vers un monde mythifié, harmonieux, sans souffrances et sans conflits, est le contrepoids d'un monde réel dévasté par le pouvoir autoritaire, la pauvreté et les inégalités sociales.

Le paroxysme est atteint sous la dictature des colonels (1967-1974), régime militariste d'un nationalisme extrême. Les manuels scolaires de lecture et de géographie ne se contentent plus de fournir des descriptions idéalisantes de la Grèce ; ils exaltent aussi l'œuvre du gouvernement dans le domaine du développement économique. Citons deux passages de manuels de géographie pour le primaire :

L'effort constant $\mathrm{du}$ Gouvernement national pour industrialiser le pays, restructurer les cultures et réaliser de grands travaux productifs aura pour conséquence la hausse du revenu par tête et la prospérité du peuple grec ${ }^{22}$.

La Grèce est un pays agricole [...] la production nationale a rapidement augmenté. Nous avons atteint l'autosuffisance en blé et le monde rural connaît des jours de prospéritées

Par cette image d'une Grèce heureuse et riche, guidée par un gouvernement éclairé, le régime dictatorial cherche à légitimer son pouvoir arbitraire. L'enseignement devient un domaine de propagande et les manuels scolaires, monopole du pouvoir central, sont mis au service du régime. L'exagération conduit à la caricature :

Les habitants, aujourd'hui gais et en bonne santé, travaillent sur leurs terres du matin au soir et ne cessent d'être reconnaissants envers l'État qui n'a pas été avare d'argent et de peine pour les rendre heureux ${ }^{24}$.

Même dans le cas où la Grèce n'est pas présentée comme un pays prospère dans tous les domaines, même lorsqu'on laisse entendre que des problèmes existent, on le fait sur un mode optimiste, ou on évite de se référer aux causes :

[Athènes] est une ville qui a sans doute ses problèmes et ses imperfections mais qui n'a rien à envier à une autre grande ville du monde. [...] Chez nous, on peut constater que la population rurale émigre vers les grandes villes. La raison qui force les paysans à partir est surtout l'insuffisant rendement des terres ${ }^{25}$.

27 Les livres de lecture des années soixante et soixante-dix s'efforcent ainsi de dissimuler la réalité sociale et de donner une impression d'harmonie et de bonheur collectif. Cette harmonie est à l'image d'une Grèce rurale qui fournit l'ensemble de la matière de ces manuels. La société rurale traditionnelle est ainsi implicitement le modèle de l'organisation sociale, du travail « noble » et des valeurs collectives « pures ».

Cette image de la Grèce rurale, voire de la Grèce entière, véhiculée par les manuels de lecture après la fin de la guerre civile présente une remarquable cohérence idéologique et elle s'est perpétuée, du fait de rééditions successives, pendant une vingtaine d'années ${ }^{26}$. Aussi ces livres n'ont-ils plus aucun rapport avec la société grecque qui subit alors des transformations rapides (c'est la période de la plus forte urbanisation), et avec la vie des élèves. Par exemple, le livre de lecture de la deuxième année de primaire publié en 1979 (19e édition) conseille aux élèves de veiller à ne pas être piétinés par des 
chevaux (!), et il les représente en train d'acheter leurs cahiers chez l'épicier contre trois œufs ${ }^{27}(!)$.

Le paysan, personnage central du récit, et son travail, condensent les valeurs dominantes que l'enseignement se propose d'inculquer aux jeunes générations. Comme l'écrit Anna Frangoudaki, «le paysan est présenté de façon sentimentale et idyllique. Son labeur accompli à la sueur de son front, en toute honnêteté, est décrit en relation esthétique avec le paysage, la terre, le soleil couchant et la cloche lointaine qui sonne les vêpres. C'est une figure noble, proche de la nature et donc de la pureté originelle, enviable du fait de son bonheur tranquille. Si les livres de lecture étaient notre seule source d'information, nous pourrions croire que le paysan est le plus heureux des hommes $^{28}$ ». Les travaux agricoles sont d'ailleurs l'occasion d'une fête continue ; il n'y a ni fatigue ni peine dans la vie des paysans grecs : « Les vendanges sont une longue fête. [...] On n'entend toute la journée que des voix gaies, des chansons, des rires ${ }^{29} . . . »$

Dans ce monde rural, la femme travaille avec les hommes aux champs, mais elle s'occupe surtout du ménage. Du fait de l'absence totale de la Grèce urbaine dans les manuels scolaires, la représentation de la famille et du rôle des femmes est une pure fiction. On lit dans le livre de lecture de la deuxième année de primaire :

Les mains de la grande-mère ne cessent de tricoter. Elle tricote des chaussettes épaisses pour le grand-père, ses fils et ses petits-enfants. La grande-mère est très habile. Ses belles-filles tissent des tapis, des couvertures... Aussi, quand l'hiver arrive, rien ne manque chez Lazaris. C'est une maison bien tenue ${ }^{30}$.

31 Le village, le champ et la petite maison villageoise sont donc les lieux autour desquels se construit le monde des livres de lecture, voire le monde des élèves de primaire. Si le livre de lecture peut être considéré comme l'abrégé du savoir fourni par l'école primaire, les enfants grecs n'apprennent presque rien sur le monde urbain jusqu'à la fin des années soixante-dix. Les expériences quotidiennes de la moitié des enfants scolarisés ${ }^{31}$, les occupations de leurs parents, les voitures, la télévision, le cinéma, le paysage urbain ne franchissent pas le seuil de l'école. Même l'image de la Grèce rurale ne traduit en rien les transformations apportées par la technologie et la machine. Le monde rural semble immobile depuis des siècles, protégé de toute altération. Il semble que sa valeur symbolique augmente en rapport direct avec l'éloignement de la réalité.

Dans les années quatre-vingt et quatre-vingt-dix : nostalgie contre progrès

Les années quatre-vingt représentent une coupure profonde dans le contenu de l'éducation en général et dans celui des manuels scolaires en particulier. Un changement se préparait depuis la chute de la dictature des colonels (1974) par la démocratisation graduelle des institutions, mais il a fallu attendre la réforme de l'éducation de 1976 (introduction officielle de la langue parlée dans l'enseignement) ${ }^{32}$ et la rédaction de nouveaux curricula et de nouveaux manuels. Contrairement à la période précédente (1949-1974), celle-ci, qui court jusqu'à nos jours, est marquée par des changements relativement fréquents des manuels visant à actualiser leur contenu. Les manuels deviennent ainsi plus proches de la réalité sociale.

Les livres de lecture des années quatre-vingt sont donc situés en milieu urbain. Le livre de lecture à l'usage des élèves de la troisième année de primaire (première édition en 1979) se situe à Athènes ${ }^{33}$. L'héroïne principale, la petite Myrto, vient d'un village grec et n'aime pas la grande ville. Ses parents ont émigré en Allemagne et elle vit chez ses tantes. Dans ce livre la comparaison entre le village et la ville, entre la campagne et Athènes, est constante. Le village est presque idéalisé grâce aux descriptions 
nostalgiques de Myrto, ce qui incite les enfants à entreprendre des travaux d'embellissement de leur quartier. Toutefois, la présentation «dialectique » de la campagne et de la grande ville ne remet pas en question la prépondérance du monde urbain. Le système de référence des enfants est construit sur la ville; le village évoque un monde beau mais lointain. Parallèlement, les changements survenus dans la production agricole et la vie rurale sont évoqués. L'idéalisation nostalgique du village ne signifie pas l'exaltation du mode de vie traditionnel.

Autrefois les protagonistes du labourage étaient la charrue, les bœufs et la sueur humaine. Aujourd'hui, c'est le tracteur avec ses dents d'acier. Autrefois, on labourait en chantant. Aujourd'hui, on n'entend que le bruit de la machine. Mais à cette époque, les hommes se fatiguaient au-delà de leurs forces et, le soir venu, ils se couchaient épuisés. Tandis qu'aujourd'hui le travail se fait de manière moins fatigante, plus facile et plus systématique ${ }^{34}$.

Le livre de lecture de la cinquième classe, publié aussi en 1979, exprime une attitude semblable, bien qu'il soit conçu selon le modèle des textes indépendants, qui ne sont pas tous contemporains :

Je regarde avec plaisir la machine conquérir les plaines de Macédoine. Et je songe que le travail se fait maintenant beaucoup plus vite, beaucoup mieux et de façon plus humaine. Que soient bénies les patientes bêtes qui pendant des siècles ont inscrit avec leurs pattes le même cercle dans l'aire. Mais que soit béni plus encore l'esprit inventif qui a brisé pour toujours ce cercle inhumain ${ }^{35}$ !

Dans la description de la Grèce rurale, le travail de la terre perd donc la valeur idéologique qu'il avait jusqu'alors dans les manuels scolaires. La mécanisation de la production, la modernité et le progrès technique se substituent à l'univers immobile du monde traditionnel. Le changement politique de 1974 semble secouer l'attachement à un monde dépassé, symbole de valeurs traditionnelles et conservatrices. La confiance dans le "changement» (qui devient d'ailleurs le slogan principal du nouveau parti socialiste PASOK, vainqueur des élections en 1981) transparaît dans les mentalités.

Le « changement » dans l'éducation se marque dans une révision profonde du contenu des manuels scolaires et de leur message idéologique. Mais la modernisation des manuels de géographie est beaucoup plus radicale que celle des livres de lecture. Nous pourrions supposer que cette particularité est due au fait que ces manuels enseignent une science, et qu'ils sont ainsi davantage susceptibles d'intégrer la modernité et la réalité contemporaine que les livres de lecture. Dans les années quatre-vingt, ces manuels scolaires de géographie font ainsi mention des changements survenus dans la Grèce rurale et des différents paramètres de l'urbanisation. L'importante diminution de la population rurale en faveur de la population urbaine et la modernisation de l'agriculture qui améliore et accélère la production sont les deux pôles du discours géographique sur la Grèce rurale. Les conséquences de l'exode rural et de l'urbanisation sont observées dans les campagnes comme dans les villes. La campagne connaît une diminution continuelle de sa population et un marasme économique, alors que l'âge moyen de la population rurale s'élève. Pour la Grèce urbaine, on évoque le gigantisme d'Athènes et de Thessalonique, les problèmes du logement, l'excès de l'offre de maind'œuvre, les emplois parasitaires, la société de consommation ${ }^{36}$. Athènes en particulier est censée être une grande ville européenne avec ses avantages et ses inconvénients : embouteillages, pollution, absence de plan d'urbanisme rationnel ${ }^{37}$...

Les manuels de géographie actuellement utilisés dans le primaire et le secondaire ne s'occupent que très peu de géographie politique. Leurs sujets s'ordonnent selon de 
grandes rubriques thématiques de géographie physique et économique : rapports de l'homme avec la nature et l'environnement, climat et topographie, activités humaines et vie sociale, démographie et économie, évolution et changements dans la vie des hommes d'hier à aujourd'hui. La Grèce n'est pas étudiée en tant que telle, mais les exemples fournis sont généralement empruntés au cas grec qui est familier aux élèves ${ }^{38}$. Les manuels de géographie contemporains attestent la confiance dans la modernisation et le progrès, c'est-à-dire en fin de compte dans la civilisation contemporaine et la raison humaine ${ }^{39}$.

Les descriptions du village et de la ville n'ont rien de commun avec celles rencontrées auparavant, à l'exception des manuels des années quatre-vingt. De ces années quatrevingt aux années quatre-vingt-dix, on observe une continuité, mais aussi une nette amélioration du contenu et de la forme. Le village n'est plus un lieu idyllique où règnent les valeurs traditionnelles, où les hommes travaillent la terre du matin au soir et où les femmes tricotent à la maison. "Un village grec », décrit dans le livre d'étude de l'environnement de la quatrième classe de primaire, est illustré par six photos : l'olivaie, la coopérative agricole, la rue principale, le collège, le centre médical et l'association culturelle ${ }^{40}$. Les pôles de la vie rurale ne sont plus le champ, l'église, l'école primaire, l'humble maison où la famille se rassemble. La production agricole est gérée par la coopérative, la vie quotidienne ne se limite pas à la maison ou au café pour les hommes, les gens peuvent s'instruire et être soignés sur place. Les principaux acteurs du village ne sont d'ailleurs plus l'instituteur et le prêtre; ce sont les membres du conseil de la commune qui s'occupent des problèmes de leur région. Les manuels scolaires insistent aussi sur l'importance de la coopérative agricole pour l'amélioration de la production, l'expansion des cultures et l'offre d'emplois. Dans la deuxième partie du même manuel d'étude de l'environnement, les activités et les bienfaits de la coopérative sont décrits de la façon suivante :

En deux ans elle a augmenté la production, les ventes (sur le marché national et à l'exportation) et le profit [...] Elle a donné du travail pour deux mois à 200 ouvriers et ouvrières [...] Elle compte étendre les cultures [...] Elle répond à divers besoins des adhérents, organise des activités culturelles, etc. ${ }^{41}$.

Le même livre prône d'ailleurs l'interventionnisme de l'État dans le domaine de l'économie rurale :

L'État montre un intérêt particulier pour le développement de l'agriculture et la protection des agriculteurs. Il intervient de plusieurs façons pour que notre pays produise plus et mieux, de façon à couvrir nos besoins et à pouvoir exporter. Par le biais de divers services publics, il programme et met en œuvre des travaux pour la modernisation de l'agriculture. Il conseille et forme les agriculteurs, à l'aide de scientifiques spécialisés dans de nouvelles méthodes de culture. Il s'occupe de la vente des produits à bon prix, accorde des subventions pour les cultures, et des indemnités en cas de catastrophes ${ }^{42}[. .$.

Les élèves sont aussi invités à expliquer et à comprendre les changements de la structure démographique de la Grèce. Par exemple, pour les rapports entre population rurale et population urbaine, les manuels scolaires comparent l'évolution des effectifs dans différentes régions (rurales, semi-urbaines et urbaines) afin d'interpréter l'évolution spécifique à chaque cas. La diminution de la population rurale est directement ou indirectement attribuée à la mécanisation de la production agricole ${ }^{43}$. Dans le manuel de géographie de la première année de collège, les causes sont plus nuancées : 
Plus la population rurale augmentait, plus la surface cultivable par agriculteur diminuait. Parallèlement, la technologie améliorait continuellement les outils et les méthodes de culture et la terre requérait moins d'hommes. Aussi nombre de paysans sans terre et sans travail ont-ils été dans les villes qui offraient plusieurs alternatives. Ils pouvaient y devenir artisans et échanger leurs produits (mobilier, vêtements, chaussures, ustensiles divers...) contre tout ce dont ils avaient besoin. Ils pouvaient aussi trouver à s'occuper dans le commerce, parce que là où réside une importante population, il y a une plus grande demande de biens. Par ailleurs, les villes étaient sûres et elles offraient des loisirs et de meilleurs services. Toutes choses qui rendaient la vie urbaine plus agréable que la vie rurale ${ }^{44}$.

41 L'univers décrit par les manuels scolaires contemporains est indéniablement l'univers familier des élèves, proche de leurs expériences quotidiennes en famille et dans le quartier, univers qu'ils connaissent aussi par les médias - surtout par la télévision. Dans le manuel le plus récent, on comprend que la plupart des élèves habitent désormais dans un grand centre urbain, et en particulier à Athènes. L'urbanisation démesurée de la région athénienne est même un des principaux thèmes de la description du pays. Les enfants sont invités à en énumérer les causes, mais aussi les conséquences ${ }^{45}$. Les exercices qui correspondent à chaque chapitre sont en effet destinés à aider l'élève à appliquer le savoir théorique à son vécu quotidien. Voici par exemple les «activités » des élèves à la fin du chapitre "Problèmes des grandes villes » :

[...] b) Étudie les annonces dans les journaux et note les écarts entre le loyer d'un appartement de cinq pièces $\left(110-140 \mathrm{~m}^{2}\right)$ situé au centre de la ville, dans un des quartiers considérés comme privilégiés, et dans la banlieue ; c) Discute avec tes parents du temps qu'ils mettent chaque jour pour aller à leur travail et en revenir. Calcule ensuite en pourcentage de combien leur temps de travail quotidien est augmenté du fait du temps qu'ils passent dans les transports ${ }^{46}[\ldots]$

La vie dans la grande ville n'est pas idéalisée. Dans les livres de lecture des deux dernières décennies, la vie des enfants dans des appartements est même souvent présentée comme triste et contraignante :

À Athènes, les enfants habitent dans des immeubles. Ils ne peuvent pas courir parce qu'ils gênent ceux qui habitent à l'étage du dessous. Ils ne peuvent pas crier parce qu'ils gênent ceux qui habitent en dessous et au-dessus. [...] L'air est pollué du fait des fumées et des gaz d'échappement des voitures. Et pour voir un peu de verdure ou se promener au bord de la mer, on doit partir en excursion ${ }^{47}$.

Les manuels scolaires contemporains reflètent donc de façon plus ou moins précise la physionomie moderne de la Grèce, tout à la fois rurale et urbaine. La Grèce rurale semble y avoir deux faces: l'une moderne, marquée par le progrès technique et la modernisation de la vie paysanne, et l'autre, mélancolique, se référant au paradis perdu d'une vie simple et heureuse près de la nature. Ces deux aspects peuvent être tout à la fois contradictoires et complémentaires. La contradiction réside dans le fait que la Grèce rurale est décrite tantôt comme abandonnée, tantôt comme prospère. La Grèce abandonnée est la Grèce traditionnelle. De façon caractéristique, par exemple, les textes cités au début de cet article, qui évoquent le village déserté ou le village comme lieu de villégiature, se réfèrent à l'image du monde rural traditionnel. Le village abandonné a les petites maisons, les rues étroites, l'église et l'école que l'on a rencontrées dans l'analyse des manuels de la période précédente (1949-1974). En revanche, le message moderniste avancé surtout par les manuels scolaires de géographie renvoie à une campagne développée, où la vie, comparativement au passé, s'est améliorée. Le visage moderne et le visage traditionnel de la campagne grecque ne 
sont pourtant pas nécessairement contradictoires. Il est probable que deux ou plusieurs réalités, a priori contradictoires, coexistent. En fait, l'exode rural n'a pas affecté toutes les régions avec la même intensité, tout comme la mécanisation de la production agricole et la modernisation de la vie des paysans n'ont pas touché toute la Grèce rurale. La mélancolie du village abandonné par ses habitants et l'activité de la coopérative agricole sont donc les deux versants d'une réalité équivoque.

L'analyse rétrospective et comparative des manuels scolaires depuis la première guerre mondiale montre ainsi que l'image de la réalité sociale varie selon le moment historique. Le discours axiomatique et édifiant des manuels va à l'encontre d'une description précise et immédiate des changements politiques, économiques et sociaux. Mais le rapport - ou l'écart - entre la réalité et sa représentation est fonction du régime politique, de l'idéologie dominante et du rôle attribué à l'éducation, contrôlée par l'État. Les deux ruptures importantes de l'histoire grecque du XXe siècle - la Discorde nationale de l'entre-deux-guerres et la Guerre civile d'après-guerre - qui ont abouti toutes deux à des dictatures, n'ont pas eu les mêmes effets sur le contenu des manuels. Malgré leurs palinodies, les manuels scolaires de l'entre-deux-guerres sont imprégnés d'un réalisme optimiste et d'une confiance dans le progrès. Ils professent donc la modernisation de la Grèce rurale et l'industrialisation du pays. En revanche, les manuels scolaires d'après-guerre retournent au rêve d'une Grèce rurale traditionnelle qui condense les valeurs primordiales du caractère national. Il faut connaître cette image idéalisée de la campagne grecque véhiculée par les manuels pendant trois décennies pour évaluer comparativement l'image correspondante des manuels contemporains. La Grèce rurale sous sa forme traditionnelle est donc glorifiée sous le régime autoritaire imposé après la fin de la guerre civile et jusqu'à la chute de la dictature militaire. Cette idéalisation passéiste aide à faire silence sur les réalités gênantes et à se réfugier dans le passé. Le rétablissement du régime parlementaire (1974) et la montée au pouvoir du parti socialiste (1981), qui absorbe une grande partie de la gauche, remettent la confiance dans le présent à l'ordre du jour et permettent une réconciliation graduelle avec le passé récent. Les manuels scolaires, toujours sous le contrôle du pouvoir central, reflètent ce tournant dans les mentalités dominantes. La Grèce rurale n'est plus un lieu de rêverie passéiste; désormais, elle acquiert sa place réelle dans la société grecque contemporaine.

\section{NOTES}

1. Nous et le monde. Étude de l'environnement (en grec), Athènes, Office d'édition des livres scolaires, 1996 (10éd.), p. 192-194.

2. L'enseignement grec comporte trois cycles : l'enseignement primaire qui dure six ans, l'enseignement secondaire (trois ans de gymnase et trois ans de lycée) et l'enseignement supérieur. L'enseignement obligatoire dure neuf ans.

3. Ma langue pour la deuxième année de l'école primaire. Seconde partie (en grec) Athènes, Office d'édition des livres scolaires, 1996 (14 éd.), p. 49. 
4. Ma langue, pour la cinquième année de l'école primaire. Quatrième partie (en grec), Athènes, Office d'édition des livres scolaires, 1991 ( $8^{\mathrm{e}}$ éd.), p. 50.

5. Ma langue, pour la deuxième année de l'école primaire... (en grec), op. cit., p. 17. Cf. aussi Ma langue, pour la quatrième année de l'école primaire. Première partie (en grec), Athènes, Office d'édition des livres scolaires, 1997 (14 éd.), p. 6.

6. Les manuels scolaires sont rédigés par des auteurs nommés par le ministère de l'Éducation et publiés par un service public, l'Office d'édition des livres scolaires (OELS).

7. M. Chouliarakis, Évolution de la population des régions rurales de la Grèce, 1920-1981 (en grec), Athènes 1988.

8. P. Tsachouridis, «L'exode rural et la modification du profil démographique de la campagne grecque » (en grec), Le Monde rural dans l'Aire méditerranéenne. Actes du Congrès franco-hellénique d'Athènes, 4-7 déc. 1984, Athènes, 1988, p. 215-237.

9. Nous avons dépouillé tous les livres de lecture et les manuels scolaires de géographie actuellement utilisés dans les établissements de l'enseignement primaire et secondaire. Pour les périodes antérieures, du fait du nombre considérable de manuels, nous avons préféré analyser un échantillon, en essayant de couvrir toutes les sous périodes établies par l'historiographie grecque.

10. G. Kazantzaki, Le jeune agriculteur. Livre de lecture de la quatrième classe des écoles primaires (en grec), Athènes 1921 (première édition en 1914), p. 3-4. La plupart des livres de lecture des années vingt sont des rééditions de manuels publiés après 1913 c'est-à-dire après les nouveaux programmes de l'école primaire introduits par le gouvernement de Vénizélos. Un cas particulier est constitué par les livres de lecture en langue parlée (démotiki) publiés après la réforme de l'éducation de 1917, qui ont provoqué de vives réactions et ont été bannis des écoles en 1920.

11. Voir M. Vitti, Fonction idéologique de l'éthographie grecque (en grec), $2^{\mathrm{e}}$ éd., Athènes 1980 , surtout p. 68-75.

12. Ibid., p. 74.

13. D. Dimitrakos, Le monde. III partie. En voyageant dans notre patrie. Géographie de la Grèce. Atlas (en grec), Athènes [1927], p. 88. Cf. aussi G. Kazantzaki, Le soldat. Livre de lecture de la cinquième année de l'école primaire (en grec), $6^{\mathrm{e}}$ éd., Athènes 1927, p. 145-6 ; id., Le jeune agriculteur... (en grec), op. cit. ; id., La Grande Grèce. Livre de lecture de la sixième année de l'école primaire (en grec), $6^{\mathrm{e}}$ éd., Athènes 1927, p. 107.

14. G. Th. Mavrogordatos, «Vénizélisme et modernisme bourgeois », in G. Th. Mavrogordatos-Ch. Hadjiossif (ed.), Vénizélisme et modernisme bourgeois (en grec), Iraklio 1988, p. 17.

15. Voir par ex. N. Ambatzoglou-D. Loukopoulos, $\mathrm{Zv}_{\text {, }}$ La vie. Livre de lecture en langue katharevoussa de la sixième année de l'école primaire (en grec), Athènes 1932 ; N.E.

Franghiskos-N.I. Protopapas, Livre de lecture pour la cinquième année de l'école primaire (en grec), Athènes 1936 ; I. Arvanitakis, Livre de lecture de la sixième année (en grec), Athènes

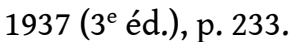

16. .Voir A. Liakos, Travail et politique en Grèce dans l'entre-deux-guerres. L'Office International du Travail et l'émergence des institutions sociales (en grec), Athènes 1993, p. 55-56, 411 .

17. Ibid., p. 85-87, 276.

18. G.P. Koutras-B. Georgiou, La Grèce. Géographie de la troisième et de la quatrième année de l'école primaire (en grec), Athènes, 1947, p. 15.

19. Gr. Lazarakis-Georgopoulos-A Gonos-M.Pangalos Géographie-Atlas de la Grèce. Troisième et quatrième années de l'école primaire (en grec) Athènes, 1952, p. 28. 
20. V. Petrounias-F. Kolovos, S. Sperantsas-A. Metallinos, Livre de lecture de la troisième année de l'école primaire (en grec), Athènes, OELS, 1954, p. 160-1. Cf. aussi Ch.

Dimitrakopoulos, Travail et joie. Livre de lecture de la deuxième année de l'école primaire (en grec), $3^{\mathrm{e}}$ éd., Athènes, OELS, 1952, p. 129.

21. N. Svoronos, Histoire de la Grèce moderne, tr. grecque de C. Asdrachas, $6^{\mathrm{e}}$ éd., Athènes, 1982, p. 147.

22. I. Gerimoglou, Géographie pour la cinquième année de l'école primaire (en grec), $2^{\mathrm{e}}$ éd., Athènes, OELS, 1970, p. 31.

23. N.M. Katsikas,) Géographie à l'usage des élèves de la sixième année des écoles primaires (en grec), $2^{\mathrm{e}}$ éd., Athènes, OELS, 1970, p. 61. Il est vrai que la production agricole avait doublé entre 1952 et 1963 et que la Grèce était autosuffisante en blé depuis 1958. Cela ne signifiait pas que le pays était prospère : le chômage augmentait, ainsi que les inégalités dans la répartition des richesses nationales, et le taux d'émigration était égal à $7 \%$ de la population du pays entre 1955 et 1964. Voir G. N. Gianoulopoulos,) Le monde de l'après-guerre. Histoire de la Grèce et de l'Europe (en grec), Athènes, 1992, p. 367-370. 24. G. Kalamatianos-Th. Gianopoulos-D. Doukas-D. Delipetros-N.Kontopoulos, Livre de lecture de la cinquième année de l'école primaire (en grec), Athènes, OELS, 1971, p. 159. 25. El. D. Mariolakos, Géographie de la Grèce (en grec), $5^{\mathrm{e}}$ éd., Athènes, OELS, 1974, p. 75 et 207. Dans le manuel de géographie de la troisième et de la quatrième classe de l'école primaire, publié pour la première fois en 1972, l'image de la Grèce rurale est cependant plus réaliste. On y fait mention de différents problèmes dans les villages : absence d'électricité et de transport, insuffisance et stérilité des terres, émigration des paysans. Néanmoins, le message optimiste ne manque pas à la fin. Ar. Chr. Kostopoulos - Victoria Kotoulopoulou-Kostopoulou, Géographie de la troisième et de la quatrième année de l'école primaire (en grec), $2^{\mathrm{e}}$ éd., Athènes, OELS, 1973, p. 39-40. Dans le même manuel, la description d'Athènes est clairement « touristique » (p. 118).

26. Les livres de lecture publiés dans les années cinquante (autorisés en 1954) furent utilisés jusqu'à la fin des années soixante-dix, à l'exception des années 1965-66. Pour une analyse de ces livres voir in A. Frangoudaki, Les livres de lecture de l'école primaire. Contrainte idéologique et violence pédagogique, Athènes 1979.

27. V. G. Oekonomidis, Livre de lecture de la $2^{e}$ année de l'école primaire (en grec), Athènes, OELS, 1979, p. 21 et 104.

28. A. Frangoudaki, op. cit., p. 71.

29. E. P. Fotiadis-El. P. Miniatis-G. Megas-D. Oekonomidis-Th. Paraskevopoulos, Livre de lecture de la sixième année de l'école primaire (en grec), 11 éd., Athènes, OELS, 1972, p. 168.

30. V. G. Oekonomidis, op. cit., p. 102.

31. En 1970-1, la proportion des élèves de l'enseignement primaire dans des régions urbaines s'élève à 44,6 \% et celle des régions rurales à 42,9\%. En 1979-80, les taux correspondants sont de $56,96 \%$ pour les régions urbaines et de 30,70\% pour les régions rurales. Office National de Statistique, Statistiques de l'Enseignement de Grèce.

32. Sur l'importance de la question de la langue pour l'identité nationale grecque, la bibliographie est abondante. Voir notamment Chr. Koulouri, « Entre l'Orient et l'Occident : les avatars de l'identité nationale grecque », Historiens et Géographes, 366, févr. 1999, p. 4.

33. A. Varella, Livre de lecture de la troisième année de l'école primaire (en grec), $2^{\mathrm{e}}$ éd., Athènes, OELS, 1980.

34. Ibid, p. 44-45. Cf. Nous et le monde. Étude de l'environnement. Troisième classe (en grec), $14^{\mathrm{e}}$ éd., Athènes, OELS, 1997, p. 30-31, 169. 
35. Th. A. Giannopoulos, Livre de lecture pour la cinquième année de l'école primaire (en grec), $4 e$ éd., Athènes, OELS, 1982, p. 203-4. On retrouve le même passage dans Nous et le monde. Étude de l'environnement. Quatrième classe. Deuxième partie (en grec), $13^{\mathrm{e}}$ éd., Athènes, OELS, 1997, p. 63.

36. Voir El. D. Mariolakos- Chr. I. Sideris, Éléments de géographie générale de la Grèce, troisième année de l'école secondaire (en grec), $5^{\mathrm{e}}$ éd., Athènes, OELS, 1983, p. 42.

37. Ibid., p. 46-7 ; Ar. Chr. Kostopoulos-V. Kotoulopoulou-Kostopoulou, La Grèce. Géographie de la quatrième année de l'école primaire (en grec), $3^{\mathrm{e}}$ éd., Athènes, OELS, 1981, p. 36 .

38. La Grèce occupe la plus grande place dans le manuel de la quatrième année de l'école primaire : Nous et le monde, op. cit. ( $1^{\text {re }}$ et $2^{\mathrm{e}}$ partie).

39. Voir l'analyse des manuels de géographie faite par L. Venturas, « Les manuels scolaires de géographie : des contradictions dans le message humaniste et moderniste » (en grec) in A. Frangoudaki-Thalia Dragonas (éd.), Qu'est-ce que notre patrie?

L'ethnocentrisme dans l'enseignement (en grec), Athènes 1997, p. 401-441. L'analyse s'appuie sur les manuels scolaires de géographie de la première, troisième et sixième années de l'école primaire et ceux de la première et troisième années du collège. Entre temps le manuel de la première année du collège a été remplacé par un nouveau manuel publié pour la première fois en 1997. En 1998 a d'ailleurs été publié un nouveau manuel de géographie pour la deuxième année du collège.

40. Nous et le monde... (en grec), op. cit., $1^{\text {re }}$ partie, p. 10-12. Cf. aussi la représentation du village incluse dans le manuel correspondant de la troisième année de l'école primaire qui comporte les points de repère suivants : l'église, l'école, l'aqueduc, l'épicerie, le café, la place, la gare des autobus, la poste et le bureau communal. Nous et le monde (en grec), op. cit., p. 8.

41. Nous et le monde..., op. cit., $2^{\mathrm{e}}$ partie, p. 64.

42. Ibid.

43. Ibid., p. 63. Beaucoup moins précise est la justification offerte par le manuel de géographie de la sixième année de primaire : «Les gens se sont déplacés vers les villes pour trouver du travail et s'assurer de meilleures conditions de vie ",) Notre Terre. Géographie de la sixième classe (en grec), $7^{\mathrm{e}}$ éd., Athènes, OELS, 1997, p. 83.

44. A. Karabatsa - A. Klonari - K. Koutsopoulos - K. Maraki - Th. Tsounakos, Géographie de la première année du collège (en grec), Athènes, OELS, 1997, p. 155

45. Nous et le monde (en grec) op. cit., $1^{\text {re }}$ partie, p. 69-72.

46. A. Karabatsa et al., op. cit., p. 166.

47. G. Grigoriadou-Soureli, Livre de lecture de la quatrième année de l'école primaire (en grec), $3^{\mathrm{e}}$ éd., Athènes, OELS, 1981, p. 194-5. Cf. aussi ? Ma langue pour la sixième classe de l'école primaire. $3^{e}$ partie (en grec), $7^{e}$ éd., Athènes, OELS, 1991, p. 35, 45 ; Ma langue pour la quatrième classe de l'école primaire. $1^{\text {re }}$ partie (en grec), 14e éd., Athènes, OELS, 1997, p. 16-17. 


\section{RÉSUMÉS}

En analysant le contenu des manuels scolaires de lecture et de géographie depuis la première guerre mondiale, l'auteur tente de montrer comment les changements démographiques, économiques et sociaux survenus en Grèce sont reflétés dans le discours scolaire. Trois grandes périodes peuvent être distinguées: la période de l'entre-deux-guerres, caractérisée par un optimiste réaliste, la période de l'après-guerre, marquée par une forte idéalisation de la Grèce rurale, censée être la gardienne des valeurs primordiales du caractère national, et la période qui va de 1974 à nos jours, où les manuels véhiculent des attitudes ambivalentes vis-à-vis de la Grèce rurale : s'y mêlent la nostalgie du paradis perdu d'une vie simple et pure près de la nature, et la confiance dans le progrès technique qui entraîne la modernisation de l'agriculture et de la vie quotidienne des paysans.

Through analysis of the content of textbooks in reading and geography since the First World War, the author attempts to show how demographic, economic and social changes in Greece are reflected in the educational discourse. Three major periods can be distinguished: the period between wars, characterised by realistic optimism; the post-war period, marked by strong idealisation of rural Greece, which was upheld as the guardian of fundamental values in the national character; and the period post-1974, in which textbooks show ambivalent attitudes towards rural Greece - a combination of nostalgia for a lost paradise where life was simple, pure and close to nature and faith in technical progress that promotes the modernisation of agriculture and of peasant life.

\section{INDEX}

Index géographique : Grèce

Thèmes : Ville et village, Manuels scolaires

Mots-clés : Rural/urbain

\section{AUTEUR}

\section{CHRISTINA KOULOURI}

Université de Thrace 